\title{
Experiência brasileira com uso de marcapasso cardíaco artificial: resultados atuais obtidos pelo Registro Brasileiro de Marcapassos - RBM
}

\author{
Roberto COSTA*, Maria Inês de Paula LEÃO*
}

RBCCV $44205-263$

COSTA, R. \& LEÃO, M. I. P. - Experiência brasileira com uso de marcapasso cardíaco artificial: resultados atuais obtidos pelo Registro Brasileiro de Marcapassos - RBM. Rev. Bras. Cir. Cardiovasc., 10 (2): 84-89, 1995.

RESUMO: Fundamentos: Registro Brasileiro de Marcapassos (RBM) é uma base nacional de dados que tem por finalidade coletar e divulgar informaçōes concernentes aos procedimentos cirúrgicos realizados em pacientes com estimulação cardíaca artificial permanente em todo o Brasil.

Objetivo: Apresentar os resultados obtidos no período de junho a dezembro de 1994.

Casuística: No período de $1 / 6 / 94$ a $31 / 12 / 94$ foram recebidos 4696 formulários enviados por 130 hospitais e 287 médicos. Os procedimentos referiam-se a $3403(72,5 \%)$ implantes iniciais a $1053(22,4 \%)$ reoperaçōes e $240(5,1 \%)$ formulários exibiam este campo de cadastramento como dado nāo disponivel.

Resultados: Dos 3403 pacientes submetidos a implante inicial, $52,8 \%$ eram do sexo masculino e $73,7 \%$ eram da raça branca. Sintomas de hipofluxo cerebral justificaram o implante em $76,4 \%$ dos pacientes e a insuficiência cardíaca congestiva esteve presente em $85,0 \%$ dos casos, sendo que em $266(7,9 \%$ ) pacientes foi a causa principal para a operaçăo. Os achados eletrocardiográficos predominantes foram: bloqueio atrioventricular total $(57,7 \%)$; disfunção do nó sinusal $(13,9 \%)$, bloqueio AV do $2^{\circ}$ grau $(13,8 \%)$; e flütter ou fibrilação atrial com baixa resposta ventricular $(6,6 \%)$. A doença de Chagas foi a etiologia predominante $(30,4 \%)$. Implante de marcapasso ventricular foi realizado em $83,6 \%$ dos pacientes $\theta$ atrioventricular em $16,1 \%$. Dos 1053 casos de reoperaçōes informados, $50,4 \%$ ocorreram por problemas no gerador de pulsos. O tempo transcorrido entre o implante inicial e a cirurgia atual variou de 1 mês a 24 anos, com média de 7,1 anos. A susbstituição do gerador de pulsos foi informada em 917 pacientes ( $87,0 \%$ dos casos de reoperação); sendo a principal causa de troca o esgotamento por fim de vida em $65,7 \%$. A substituição de eletrodo atrial foi relatada em 21 pacientes e de eletrodo ventricular em 203 pacientes.

Conclusōes: A participação ativa de todos os membros do Departamento de Estimulação Cardíaca Artificial (DECA), com o apoio das empresas fornecedoras de marcapasso, e o respaldo do Ministério da Saúde permitiram que a implantaçāo do RBM ocorresse da forma mais tranqưila possível. Hoje se dispōe de uma estatística nacional, volumosa e confiável, sobre a cirurgia de marcapasso no Brasil. As dificuldades enfrentadas estão relacionadas, principalmente, à estrutura individual de coleta de dados e a graus variados de familiaridade com o formulário, dificuldades estas que, com o tempo, tenderão a desaparecer.

DESCRITORES: Marcapassos cardiacos, cirurgia, coleta de dados. Estimulação cardíaca artificial, marcapassos, coleta de dados. Arritmias cardiacas. Registro Brasileiro de Marcapassos - RBM.

\footnotetext{
Trabalho realizado no Departamento de Estimulação Cardiaca Artificial - DECA, da Sociedade Brasileira de Cirurgia Cardiovascular. São Paulo, SP, Brasil.

Apresentado ao $22^{\circ}$ Congresso Nacional de Cirurgia Cardiaca. Brasília, DF, 30 de março a $1^{2}$ de abril, 1995.

* Médico Coordenador do Registro Brasileiro de Marcapassos - RBM.

Endereço para correspondência: Roberto Costa. Av. Brig. Luís Antônio, 4268. Cep [011] 01402-002. São Paulo, SP, Brasil. Tel.: [011] 887-8866. Fax: (011) 885-3919
} 
COSTA, R. \& LEÃO, M. I. P. - Experiência brasileira com uso de marcapasso cardiaco artificial: resultados atuais obtidos pelo Registro Brasileiro de Marcapassos - RBM. Rev. Bras. Cir. Cardiovasc., 10 (2): 84-89, 1995.

\section{INTRODUÇÃO}

O Registro Brasileiro de Marcapassos (RBM) é uma base de dados nacional que conta com a participação do DECA, do Ministério da Saúde e dos fabricantes de marcapassos. Foi implantado a partir de $1^{2}$ de junho de 1994 e tem por objetivo coletar informações a respeito dos procedimentos cirúrgicos realizados em pacientes que utilizem a estimulação cardíaca artificial permanente $1,2,4$.

A instituição de um Registro Brasileiro de Marcapassos tem especial importância por permitir ao DECA, e a seus membros, uma fonte oficial de consulta sobre os aspectos atuais da estimulação cardíaca artificial em nosso meio, ajudando não só na comparação com dados epidemiológicos internacionais, como no direcionamento das açōes prioritárias do Departamento junto a seus associados. Um exemplo simples é o conhecimento mais apurado de todos os profissionais envolvidos com os implantes, principalmente residentes, no sentido de estimulá-los a participar mais intensamente das atividades científicas do DECA, bem como de promover, quando necessárias, ações continuadas de ensino para o aprimoramento de nossa sociedade.

\section{MATERIAL}

Foi utilizado um computador PC 486-DX2-66 para processamento dos dados colhidos por uma planilha padronizada, fornecida aos fornecedores de marcapassos, em quatro vias. A distribuição destes formulários nas embalagens dos marcapassos é obrigatória por portaria do Ministério da Saúde. Após serem preenchidos pelo médico que executou o implante de marcapasso, os formulários são encaminhados à Secretaria Executiva de Procedimentos de Alta Complexidade em Cardiologia, que os envia para processamento na secretaria do RBM.

As informações são divididas em 1) dados pessoais do paciente; 2) dados clínicos; 3) dados cirúrgicos, que contêm informações sobre os sistemas de estimulação implantados e/ou removidos e 4) fechamento do arquivo.

\section{CASUÍSTICA E MÉTODOS}

No período de $1 / 6 / 94$ a 31/12/94 foram realizados 4696 procedimentos cirúrgicos relacionados à estimulação cardíaca artificial, segundo as informações registradas nos formulários enviados por 130 hospitais e preenchidos por 287 médicos. Destes, $3403(72,5 \%)$ foram de implantes iniciais, 1053 $(22,4 \%)$ de reoperações e 240 vezes $(5,1 \%)$ foi informado dado não disponível.

\section{IMPLANTES INICIAIS}

Nos 3403 casos de implante inicial, o sexo masculino foi referido em $1790(52,8 \%)$ formulários e o feminino em $1598(47,1 \%)$. A raça era branca em $2380(73,7 \%)$, mestiça em $388(12,0 \%)$, negra em $268(8,3 \%)$ e amarela em $19(0,6 \%)$ pacientes. Dado não disponível foi informado em 159 (4,9\%) formulários.

A indicação clínica para o implante de marcapasso foi justificada por síncopes em 1477 $(43,6 \%)$, tonturas em $703(20,8 \%)$, pré-síncopes em $407(12,0 \%)$, bradicardia em $337(9,9 \%)$ e insuficiência cardíaca congestiva em $266(7,9 \%)$ pacientes (Tabela 1, Figura 1). A classificação funcional para insuficiência cardíaca congestiva (NYHA) mostrou que $368(10,9 \%)$ pacientes eram assintomáticos, $421(12,4 \%)$ apresentavam sintomas aos grandes esforços; $1570(46,4 \%)$ aos médios ou pequenos esforços e $888(26,2 \%)$ em repouso. Dado não disponível foi informado em $139(4,1 \%)$ formulários (Tabela 2).

O distúrbio do ritmo cardíaco, que justificou o implante de marcapasso, foi bloqueio atrioventricular

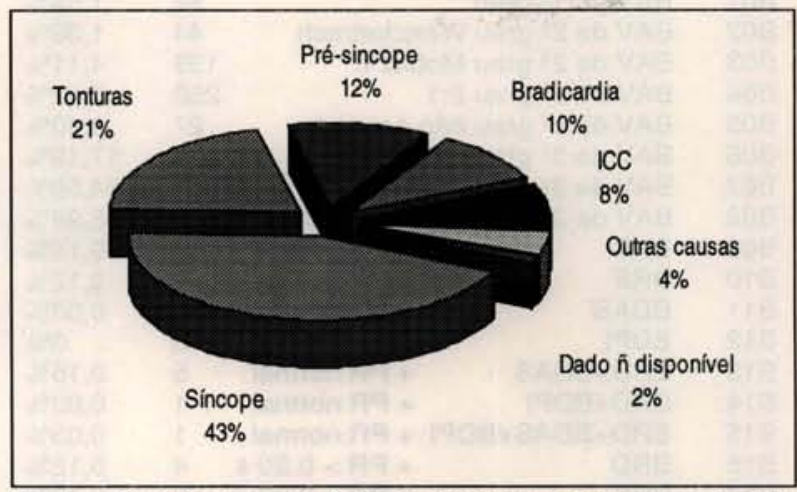

Fig. 1. Quadro clínico que indicou o implante de marcapasso inicial.

TABELA 1

QUADRO CLIINICO QUE INDICOU O IMPLANTE DE MARCAPASSOINICIAL

\begin{tabular}{llrr}
\hline Cód. & Opções & Ne & Percent. \\
\hline A01 & Síncope & 1477 & $43,62 \%$ \\
A02 & Pré-síncope & 407 & $12,02 \%$ \\
A03 & Tonturas & 703 & $20,76 \%$ \\
A04 & Insuf. Cardiaca Congestiva & 266 & $7,86 \%$ \\
A05 & Disf. Cerebral / Bradipsiq & 3 & $0,09 \%$ \\
B01 & Bradicardia & 337 & $9,95 \%$ \\
B02 & Taquicardia & 14 & $0,41 \%$ \\
B03 & Arritmia Secundária & 27 & $0,80 \%$ \\
C01 & Necessidade de fármacos & 46 & $1,36 \%$ \\
C02 & Profilático & 12 & $0,35 \%$ \\
C03 & Outras indicações não codificadas & 43 & $1,27 \%$ \\
D01 & Informação năo disponivel & 51 & $1,51 \%$ \\
\hline \multicolumn{2}{c}{ Total } & 3386 & $100,00 \%$ \\
\hline & & &
\end{tabular}


COSTA, R. \& LEĀO, M. I. P. - Experiência brasileira com uso de marcapasso cardíaco artificial: resultados atuais obtidos pelo Registro Brasileiro de Marcapassos - RBM. Rev. Bras. Cir. Cardiovasc., 10 (2): 84-89, 1995.

TABELA 2

CLASSIFICAÇÃO FUNCIONAL PARA INSUFICIÊNCIA CARDIACA CONGESTIVA DOS PACIENTES SUBMETIDOS A IMPLANTE INICIAL

\begin{tabular}{|c|c|c|c|}
\hline Cód. & Opçōes & No & Percent. \\
\hline A01 & Assintomático & 368 & $10,87 \%$ \\
\hline B02 & Grandes esforços & 421 & $12,43 \%$ \\
\hline B03 & Médios ou pequenos esforços & 1570 & $46,37 \%$ \\
\hline B04 & Repouso & 888 & $26,23 \%$ \\
\hline $\mathrm{C} 01$ & Informação não disponivel & 139 & $4,11 \%$ \\
\hline & Total & 3386 & $100,00 \%$ \\
\hline
\end{tabular}

total em $1954(57,7 \%)$, doença do nó sinusal em $272(13,9 \%)$, bloqueio atrioventricular do $2^{\circ}$ grau em $468(13,8 \%)$, e flütter ou fibrilação atrial com baixa resposta ventricular em $224(6,6 \%)$ pacientes (Tabela 3 , Figura 2). Como etiologia para o distúrbio da

TABELA 3

ACHADO ELETROCARDIOGRÁFICO QUE JUSTIFICOU O IMPLANTE DE MARCAPASSO INICIAL

\begin{tabular}{|c|c|c|c|c|}
\hline Cód. & \multicolumn{2}{|l|}{ Opçōes } & No & Percent. \\
\hline A01 & \multicolumn{2}{|c|}{ Ritmo Sinusal Normal } & 15 & $0,44 \%$ \\
\hline B01 & \multicolumn{2}{|l|}{ BAV de $1^{\circ} \mathrm{grau}$} & 52 & $1,54 \%$ \\
\hline B02 & \multicolumn{2}{|c|}{ BAV de $2^{\circ}$ grau Wenckebach } & 44 & $1,30 \%$ \\
\hline B03 & \multicolumn{2}{|c|}{ BAV de 29 grau Mobitz II } & 139 & $4,11 \%$ \\
\hline B04 & \multicolumn{2}{|c|}{ BAV de $2^{2}$ grau $2: 1$} & 258 & $7,62 \%$ \\
\hline B05 & \multicolumn{2}{|c|}{ BAV de $2^{\circ}$ grau não especif. } & 27 & $0,80 \%$ \\
\hline B06 & \multicolumn{2}{|c|}{ BAV de $3^{2}$ grau QRS Estreito } & 582 & $17,19 \%$ \\
\hline B07 & \multicolumn{2}{|c|}{ BAV de $3^{\circ}$ grau QRS Largo } & 1171 & $34,59 \%$ \\
\hline B08 & \multicolumn{2}{|c|}{ BAV de $3^{2}$ grau QRS não esp. } & 201 & $5,94 \%$ \\
\hline B09 & BRD & + PR normal & 5 & $0,15 \%$ \\
\hline B10 & BRE & + PR normal & 4 & $0,12 \%$ \\
\hline B11 & BDAS & + PR normal & 1 & $0,03 \%$ \\
\hline B12 & BDPI & + PR normal & 0 & $0 \%$ \\
\hline B13 & BRD+BDAS & +PR normal & 5 & $0,15 \%$ \\
\hline B14 & $\mathrm{BRD}+\mathrm{BDPI}$ & + PR normal & 1 & $0,03 \%$ \\
\hline B15 & $\mathrm{BRD}+\mathrm{BDAS}+\mathrm{BDP}$ & + PR normal & 1 & $0,03 \%$ \\
\hline B16 & BRD & $+P R>0,20$ & 4 & $0,12 \%$ \\
\hline B17 & BRE & $+\mathrm{PR}>0,20$ & 6 & $0,18 \%$ \\
\hline B18 & BDAS & $+\mathrm{PR}>0,20$ & 2 & $0,06 \%$ \\
\hline B19 & BDPI & $+P R>0,20$ & 0 & $0 \%$ \\
\hline B20 & $B R D+B D A S$ & $+P R>0,20$ & 24 & $0,71 \%$ \\
\hline B21 & BRD+BDPI & $+\mathrm{PR}>0,20$ & 1 & $0,03 \%$ \\
\hline B22 & $\mathrm{BRD}+\mathrm{BDAS}+\mathrm{BD}$ & $+P R>0,20$ & 8 & $0,24 \%$ \\
\hline B23 & Bloqueio Fascicul & ar não espec. & 12 & $0,35 \%$ \\
\hline B24 & Bloqueio Bilateral & Alternante & 0 & $0 \%$ \\
\hline $\mathrm{C} 01$ & Bloqueio Sinoatria & & 83 & $2,45 \%$ \\
\hline $\mathrm{CO} 2$ & Parada Sinusal & & 82 & $2,42 \%$ \\
\hline $\mathrm{CO3}$ & Bradicardia Sinus & & 209 & $6,17 \%$ \\
\hline $\mathrm{CO4}$ & Síndrome de Brad & -Taqui & 98 & $2,90 \%$ \\
\hline $\mathrm{C} 05$ & Fibril. Atrial + Bra & licardia & 199 & $5,88 \%$ \\
\hline $\mathrm{CO6}$ & Flütter Atrial + Bra & dicardia & 25 & $0,74 \%$ \\
\hline $\mathrm{CO7}$ & Disfunçāo Sinusa & nāo espec. & 0 & $0 \%$ \\
\hline D01 & Taquicardia Atrial & & 6 & $0,18 \%$ \\
\hline D03 & Extra-sistolia Ven & ricular & 3 & $0,09 \%$ \\
\hline D04 & Taquicardia Ventr & cular & 9 & $0,27 \%$ \\
\hline D05 & Fibril. Ventric. Par & oxística & 3 & $0,09 \%$ \\
\hline E01 & Outro Achado Nã & Codificado & 29 & $0,86 \%$ \\
\hline F01 & Informaçāo Nāo D & isponivel & 76 & $2,25 \%$ \\
\hline & Total & & 3385 & $100,03 \%$ \\
\hline
\end{tabular}

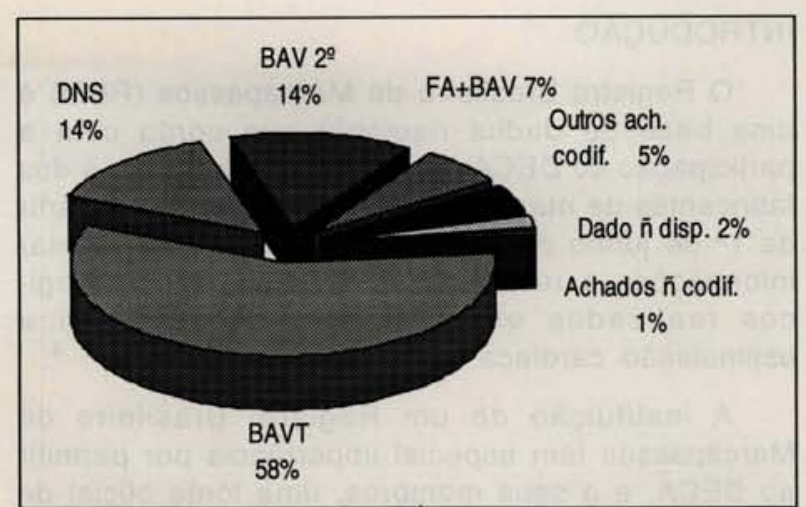

Fig. 2 - Achados eletrocardiográficos que justificaram o implante de marcapasso inicial.

condução foi considerada a doença de Chagas em $1030(30,4 \%)$, a fibrose do sistema de condução em $817(24,1 \%)$, etiologia desconhecida em $659(19.5 \%)$ e isquemia em $230(6,8 \%)$ pacientes. Dado não disponível foi referido $215(6,4 \%$ ) vezes (Tabela 4 , Figura 3).

\section{TABELA 4}

ETIOLOGIA DO DISTÚRBIO DA CONDUÇĀO QUE INDICOU O IMPLANTE DE MARCAPASSO INICIAL

\begin{tabular}{llrr}
\hline Cód. & \multicolumn{1}{c}{ Opções } & No & Percent. \\
\hline A01 & Etiologia Desconhecida & 659 & $19,45 \%$ \\
A02 & Fibrose do Sistema de Cond. & 817 & $24,11 \%$ \\
B01 & Isquemia & 230 & $6,79 \%$ \\
B02 & Pós-Infarto & 28 & $0,83 \%$ \\
C01 & Congênita & 33 & $0,97 \%$ \\
D01 & Complicação Cirúrgica & 51 & $1,51 \%$ \\
D02 & Ablação Cirúrgica & 6 & $0,18 \%$ \\
D03 & Ablação por Cateter & 17 & $0,50 \%$ \\
D04 & Uso de Fármacos & 9 & $0,27 \%$ \\
E01 & Síndrome do Seio Carotídeo & 25 & $0,74 \%$ \\
E02 & Disfunção Autonômica & 12 & $0,35 \%$ \\
F01 & Doença de Chagas & 1030 & $30,40 \%$ \\
F02 & Miocardiopatia Dilatada & 143 & $4,22 \%$ \\
F03 & Miocardite & 3 & $0,09 \%$ \\
G01 & Lesão Valvar & 20 & $0,59 \%$ \\
G02 & Endocardite & 2 & $0,06 \%$ \\
G03 & Outras Causas Não Codificadas & 88 & $2,60 \%$ \\
H01 & Informação Não Disponivel & 215 & $6,35 \%$ \\
\hline & Total & 3388 & $100,01 \%$ \\
\hline
\end{tabular}

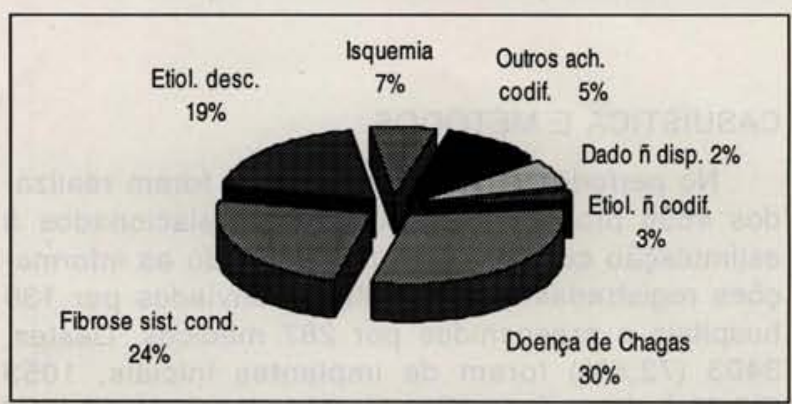

Fig. 3 - Etiologia do Distúrbio da Condução que indicou o implante de marcapasso inicial. 
COSTA, R. \& LEÃO, M. I. P. - Experiência brasileira com uso de marcapasso cardíaco artificial: resultados atuais obtidos pelo Registro Brasileiro de Marcapassos - RBM. Rev. Bras. Cir. Cardiovasc., 10 (2): 84-89, 1995.

O tipo de sistema de estimulação utilizado foi ventricular em $2843(83,6 \%)$, atrioventricular em 549 $(16,1 \%)$ e atrial exclusivo em apenas 10 pacientes ( $0,3 \%$ dos casos de implante inicial).

\section{REOPERAÇÕES}

Dos 1053 casos de reoperações informados, $531(50,4 \%)$ ocorreram por problemas no gerador de pulsos, $69(6,5 \%)$ por problemas no eletrodo, 50 $(4,8 \%)$ por aumento do limiar de estimulação, 51 $(4,8 \%)$ por distúrbios hemodinâmicos, $17(1,6 \%)$ por síndrome do marcapasso e em $12(1,1 \%)$ pacientes por estimulação frênica. Outros motivos não codificados foram referidos em 242 (23,0\%) formulários (Tabela 5).

A data do implante inicial foi informada em 830 formulários. O tempo transcorrido entre o implante inicial e o procedimento presentemente relatado variou de 1 mês a 24 anos, com média de 7,1 anos. A reoperação ocorreu antes de completar um ano do primeiro implante de marcapasso em 135 pacientes $(16,3 \%$ dos casos de reoperação), do primeiro ao quinto ano em $141(17,0 \%)$, do sexto ao décimo ano em 405 (48,8\%), do décimo primeiro ao décimo quinto ano em $124(14.9 \%)$, do décimo sexto ao vigésimo em $20(2,4 \%)$ e acima de 20 anos em $5(0,6 \%)$.

A substituição do gerador de pulsos foi informada em 917 pacientes $(87,0 \%$ dos casos de reoperação). A principal causa de troca do gerador

TABELA 5

MOTIVO PRINCIPAL PARA A REOPERAÇÃO

\begin{tabular}{llrr}
\hline Cód. & \multicolumn{1}{c}{ Opções } & Ne & Percent. \\
\hline B01 & Distúrbio Hemodinâmico & 51 & $4,84 \%$ \\
B02 & Palpitaçōes & 2 & $0,19 \%$ \\
B03 & Síndrome do Marcapasso & 17 & $1,61 \%$ \\
C01 & Dor na Ferida & 3 & $0,28 \%$ \\
C02 & Erosão da Pele & 5 & $0,47 \%$ \\
C03 & Extrusão de Sistema & 38 & $3,61 \%$ \\
C04 & Infecção & 18 & $1,71 \%$ \\
C05 & Hematoma & 2 & $0,19 \%$ \\
D01 & Problema no Gerador & 531 & $50,43 \%$ \\
D02 & Defeito do Eletrodo & 69 & $6,55 \%$ \\
D03 & Desposicionamento de Eletrodo & 2 & $0,19 \%$ \\
D04 & Aumento do Limiar & 50 & $4,75 \%$ \\
D05 & Perfuração & 2 & $0,19 \%$ \\
D06 & Alteração da Sensibilidade & 0 & $0 \%$ \\
E01 & Interferência por Miopotenciais & 6 & $0,57 \%$ \\
E02 & Interferência Eletromagnética & 3 & $0,28 \%$ \\
F01 & Estimulação Frênica & 12 & $1,14 \%$ \\
F02 & Estimulação Muscular & 0 & $0 \%$ \\
G01 & Outro Motivo Não Codificado & 242 & $22,98 \%$ \\
\hline & Total & 1053 & $99,98 \%$ \\
\hline
\end{tabular}

foi esgotamento por fim de vida em 602 pacientes ( $65,7 \%$ dos casos de troca de gerador), pela oportunidade cirúrgica em $46(5,0 \%)$, por esgotamento precoce em $34(3,7 \%)$, por queda de freqüência em $30(3,3 \%)$, pela contaminação do aparelho em 26 $(2,8 \%)$ e por solicitação do fabricante em $12(1,3 \%)$. Dado não disponível foi referido 111 vezes $(12,1 \%$ dos casos de troca de gerador) (Tabela 6).

Substituição de cabo-eletrodo foi referida em 224 casos. A troca do cabo atrial foi informada em 21 pacientes $(2,0 \%$ das reoperações) e do eletrodo ventricular em $203(19,3 \%)$. O motivo para a troca do cabo-eletrodo foi aumento do limiar em 51 pacientes, fratura do condutor em 39 , defeito do isolamento em 30 e contaminação em 29. Dado não disponível foi relatado em $39(3,7 \%)$ casos de troca de eletrodo (Tabela 7).

\section{COMENTÁRIOS}

Os autores optaram por apresentar os dados da primeira indicação cirúrgica separadamente aos das reoperações, o que permite a análise do perfil atual de indicações de marcapasso no Brasil.

Nos casos de implante inicial foi possível notar a ocorrência predominante do sexo masculino $(52,7 \%)$ e da raça branca $(73,1 \%)$. Os sintomas de hipofluxo cerebral, englobando síncopes, tonturas e pré-síncopes, justificaram $77,3 \%$ dos implantes e a

TABELA 6

MOTIVO PARA A TROCA DO GERADOR

\begin{tabular}{llrr}
\hline Cód. & \multicolumn{1}{c}{ Opções } & No & Percent. \\
\hline A01 & Oportunidade Cirúrgica & 46 & $5,02 \%$ \\
A02 & "Recall" & 12 & $1,31 \%$ \\
A03 & Problema Clínico & 7 & $0,76 \%$ \\
A04 & Interferência & 0 & $0 \%$ \\
A05 & Estimulaçao Extracardiaca & 2 & $0,22 \%$ \\
B01 & Baixa Sensibilidade & 3 & $0,33 \%$ \\
B02 & Alta Sensibilidade & 2 & $0,22 \%$ \\
B03 & Defeito no Interruptor Mag. & 1 & $0,11 \%$ \\
B04 & Falha de Programação/Telemet. & 0 & $0 \%$ \\
B05 & Defeito Menor Não Codificado & 1 & $0,11 \%$ \\
C01 & Ausência de Saída & 9 & $0,98 \%$ \\
C02 & Baixa Saída & 6 & $0,65 \%$ \\
C03 & Queda de Frequeência & 30 & $3,27 \%$ \\
C04 & Aumento de Freqūência & 3 & $0,33 \%$ \\
C05 & Defeito no Conector & 4 & $0,44 \%$ \\
C06 & Defeito na Carcaça & 0 & $0 \%$ \\
C07 & Defeito Maior Não Codificado & 0 & $0 \%$ \\
D01 & Esgotamento por Fim de Vida & 602 & $65,65 \%$ \\
D02 & Esgotamento Precoce & 34 & $3,71 \%$ \\
E01 & Contaminação & 26 & $2,84 \%$ \\
E02 & Outro Motivo Não Codificado & 18 & $1,96 \%$ \\
F01 & Dado Não Disponível & 111 & $12,10 \%$ \\
\hline & Total & 917 & $100,01 \%$ \\
\hline
\end{tabular}


COSTA, R. \& LEÃO, M. I. P. - Experiência brasileira com uso de marcapasso cardiaco artificial: resultados atuais obtidos pelo Registro Brasileiro de Marcapassos - RBM. Rev. Bras. Cir. Cardiovasc., 10 (2): 84-89, 1995.

TABELA 7

MOTIVO PARA A TROCA DO ELETRODO

\begin{tabular}{|c|c|c|c|c|c|}
\hline \multirow[b]{2}{*}{ Cód. } & \multirow[b]{2}{*}{ Opçōes } & \multicolumn{2}{|c|}{ Eletrodo Atrial } & \multicolumn{2}{|c|}{ Eletrodo Ventricular } \\
\hline & & Ne & Percent. & Ne & Percent. \\
\hline A01 & Oportunidade Cirúrgica & 2 & $9,52 \%$ & 6 & $2,96 \%$ \\
\hline $\mathrm{A} 02$ & Deslocamento do Eletrodo & 0 & $0 \%$ & 2 & $0,99 \%$ \\
\hline $\mathrm{A} 03$ & Aumento do limiar & 2 & $9,52 \%$ & 49 & $24,14 \%$ \\
\hline $\mathrm{A} 04$ & Baixa Sensibilidade & 2 & $9,52 \%$ & 6 & $2,96 \%$ \\
\hline A05 & Interferência por Miopot. & $\overrightarrow{0}$ & $0 \%$ & 2 & $0,99 \%$ \\
\hline A06 & Estimulaçāo Extracardíaca & 0 & $0 \%$ & 0 & $0 \%$ \\
\hline A07 & Protrusão Cutânea & 2 & $9,52 \%$ & 3 & $1,48 \%$ \\
\hline B01 & Defeito da Conexão & 0 & $0 \%$ & 4 & $1,97 \%$ \\
\hline B02 & Rotura do Isolamento & 5 & $23,81 \%$ & 25 & $12,32 \%$ \\
\hline B03 & Fratura do Condutor & 2 & $9,52 \%$ & 37 & $18,23 \%$ \\
\hline $\mathrm{CO}$ & Contaminação & 5 & $23,81 \%$ & 24 & $11,82 \%$ \\
\hline $\mathrm{CO2}$ & Outro Motivo Não Codificado & 0 & $0 \%$ & 7 & $3,45 \%$ \\
\hline $\mathrm{CO3}$ & Perfuração & 0 & $0 \%$ & 0 & $0 \%$ \\
\hline \multirow[t]{2}{*}{ D01 } & Dado Năo Disponível & 1 & $4,76 \%$ & 38 & $18,72 \%$ \\
\hline & Total & 21 & $99,98 \%$ & 203 & $100,03 \%$ \\
\hline
\end{tabular}

presença isolada ou em associação de insuficiência cardíaca congestiva foi relatada em $85,6 \%$ dos formulários de primeiro implante. Os bloqueios avançados da condução AV foram responsáveis por $71,4 \%$ das indicações de implante de marcapasso, ficando a doença do nó sinusal com $15,0 \%$ e flutter ou fibrilação atrial de baixa resposta ventricular com $6,7 \%$. A doença de Chagas foi a etiologia predominante $(30,0 \%)$, seguida por fibrose do sistema de condução $(25,3 \%)$ e etiologia desconhecida $(21,1 \%)$. Marcapassos ventriculares implantados em $84 \%$ dos pacientes continuam sendo a principal configuração utilizada, ficando os marcapassos de dupla câmara com apenas $16 \%$. Verificou-se, ainda, a pequena utilização da estimulação atrial exclusiva, relatada em apenas $2(0,1 \%)$ pacientes. Estes dados são fortemente semelhantes aos obtidos no trimestre junho-agosto de $1994^{3}$.

A forma como foi idealizado o formulário do RBM permite a análise das reoperações levando em consideração dois tempos distintos: inicialmente, o motivo principal para a indicação cirúrgica, e a partir da avaliação per-operatória, num segundo momento reanalisa o motivo para a troca de gerador, eletrodos ou outros procedimentos previstos ou não antes da operação.

$\mathrm{Na}$ primeira análise vê-se que, dentre os 590 casos de reoperações, $281(47,6 \%)$ foram devidos a "problemas no gerador". Chama a atenção o grande contigente de 156 casos relatados de "outro motivo não codificado" (26,4\% das reoperações) (Tabela 5). Quando se analisa a Tabela 6, que justifica o motivo para troca de gerador, vê-se que este procedimento foi diretamente imputado a defeitos do gerador em 397 casos (solicitação do fabricante, ausência de saída, queda de freqüência, defeito no conector, esgotamento por fim de vida, esgotamento precoce, dentre outros). Verifica-se, portanto, uma incongruência na classificação inicial do motivo principal para a operação, com a utilização indevida da opção "outro motivo não codificado" em lugar de "problemas no gerador". Dentre os motivos para a troca de cabo-eletrodo ventricular, destacam-se o aumento do limiar, justificando $24,6 \%$ das trocas, a fratura do condutor em $17,0 \%$ e a rotura de isolamento em $11,0 \%$.

Dificuldades de preenchimento do formulário do RBM poderiam ser atribuídas a fatores como 1) complexidade do formulário; 2) importância relativa de cada um de seus campos e 3) prática entre equipes cirúrgicas de delegar a função de preenchimento do Registro a membros não familiarizados com o caso, limitando-se apenas a dados técnicos da operação. A comparação com a estatística do trimestre anterior, entretanto, mostra uma superposição de porcentagens, principalmente no que diz respeito aos casos de primeiro implante, ressaltando a boa qualidade da coleta de informações. Os problemas acima apontados devem-se, provavelmente, não somente à interpretação das Tabelas, como também à dificuldade de obtenção, junto aos pacientes, de dados de operações anteriores.

Desde sua criação, o RBM procurou obter informações de cunho puramente científico, não tendo qualquer preocupação quanto à classificação social do paciente, ou das condições em que foram realizados os procedimentos. Isto fez com que a maioria dos colegas passasse a informar a totalidade de seu movimento, o que tem justificado o aumento progressivo dos formulários recebidos. 
COSTA, R. \& LEÃO, M. I. P. - Experiência brasileira com uso de marcapasso cardíaco artificial: resultados atuais obtidos pelo Registro Brasileiro de Marcapassos - RBM. Rev. Bras. Cir. Cardiovasc., 10 (2): 84-89, 1995.

\section{RBCCV 44205-263}

COSTA, R. \& LEÃO, M. I. P. - Brazilian permanent cardiac pacemaker implantation experience: results obtained from the Brazilian Pacemaker Register's data. Rev. Bras. Cir. Cardiovasc., 10 (2): 84-89, 1995.

ABSTRACT: Background: Brazilian Pacemaker Registry (RBM) is a nationwide database to collect informations about all permanent pacemaker procedures performed in Brazil. It is a task force composed by Medical Society, Health Ministery and Pacemaker Companies.

Objective: To report the data obtained from June to December, 1994.

Methods: From June 1st to December 31, 1994, 4696 surgical procedures for permanent cardiac pacing were informed. These procedures were $3403(72,5 \%)$ initial implantations, $1053(22.4 \%)$ re-operations and in 240 cases this information was non-available.

Results: From 3403 initial implantations informed, $52.8 \%$ were males and $73.7 \%$ caucasians. Preoperative dizziness or syncopes were refered in $76.4 \%$ and congestive heart failure in $85.0 \%$ of the patients. EKG evaluation showed 3rd degree atrioventricular (AV) block in $57.7 \%$, sick sinus syndrome in $13.9 \%, 2$ nd degree AV block in $13.8 \%$, and high degree AV block and flütter or atrial fibrillation in $6.6 \%$ of patients. Chaga's disease was the predominat ethiology $(30.4 \%)$. Ventricular pacemakers were implanted in $83.6 \%$ of cases, atrioventricular in $16.1 \%$ and atrial pacing in $0.3 \%$. In the 1053 cases of re-operation, the interval between the initial implantation and the present procedure ranged from 1 month to 24 years $(M=7.1$ years). Pulse generator replacements were refered in 917 patients ( $87.0 \%$ of re-operation procedures), $65.7 \%$ of them at the end of life. Atrial lead replacement were performed in 21, and ventricular lead replacement in 203 patients.

DESCRIPTORS: Cardiac pacemakers, surgery, data collection. Cardiac stimulation, artificial, data collection. Cardiac arrithmias. Brazilian Pacemaker Registry - RBM.

\section{REFERÊNCIAS BIBLIOGRÁFICAS}

COSTA, R. \& LEĀO, M. I. P. - Implantaçāo do Registro Brasileiro de Marcapassos. Rev. Bras. Marcapasso Arritmia, 7: 2-3, 1994.

2 COSTA, R. \& LEĀO, M. I. P. - Registro Brasileiro de Marcapassos. Rev. Bras. Marcapasso Arritmia, 6: 31-34, 1993.
3 COSTA, R. \& LEĀO, M. I. P. - Registro Brasileiro de Marcapassos: resultados preliminares. Rev. Bras. Marcapasso Arritmia, 7: 124-129, 1994.

4 LEÃO, M. I. P.; COSTA, R.; LATINI, R. - Registro Brasileiro de Marcapassos: orientação para preenchimento do formulário. Rev. Bras. Marcapasso Arritmia, 7: 72-77, 1994 